\title{
Cold stress affects antioxidative response and accumulation of medicinally important withanolides in Withania somnifera (L.) Dunal
}

\author{
Bilal Ahmad Mir ${ }^{\mathrm{a}, \mathrm{b}, *}$, Shabir Ahmad Mir ${ }^{\mathrm{c}}$, Jabeena Khazir ${ }^{\mathrm{d}}$, Libert Brice Tonfack ${ }^{\mathrm{e}}$,
} Don A. Cowan ${ }^{\text {b }}$ Dhiraj Vyas ${ }^{\text {a }}$, Sushma Koul ${ }^{\text {a }}$

\begin{abstract}
a Biodiversity \& Applied Botany Division, Indian Institute of Integrative Medicine, Canal Road, Jammu 180001, India
${ }^{\mathrm{b}}$ Centre for Microbial Ecology and Genomics \& Genomics Research Institute, Department of Genetics, University of Pretoria, Pretoria 0028, South Africa c Department of Biochemistry, University of Pretoria, Pretoria 0028, South Africa

${ }^{\mathrm{d}}$ Department of Chemistry, University of Pretoria, Pretoria 0028, South Africa

e Laboratory of Biotechnology and Environment, Unit of Plant Physiology and Improvement, Department of Plant Biology, Faculty of Science, University of Yaoundé, Yaoundé, Cameroon
\end{abstract}

\begin{abstract}
Withania somnifera (L.) Dunal (Indian ginseng) is a high value medicinal plant. It synthesizes a large array of biologically active withanolides. In this study, two month old seedlings of AGB002 (wild genotype) and AGB025 (cultivated genotype) of $W$. somnifera were subjected to cold stress ( $4{ }^{\circ} \mathrm{C}$ ) under controlled envi-ronment. Plants were analyzed for three medicinally important secondary metabolites (withanolide A, withanone and withaferin A), lipid peroxidation (MDA), cell injury, superoxide radical $\left(\mathrm{O}_{2}{ }^{-}\right)$accumula-tion and anti-oxidative enzymes activities such as superoxide dismutase (SOD), catalase (CAT), ascorbate peroxidase (APX) and glutathione reductase (GR). Increases in the titers of superoxide anion and MDA were observed from day 1 to day 7 in both genotypes, although the increase on the first day of exposure was significantly higher. Enzymatic activities of SOD, CAT, APX and GR also showed an increasing trend in both genotypes and reached a maximum on day 7 of the cold temperature exposure; however, this increase was higher in AGB002 than AGB025. Withanolide A (WS-1) in the roots of both genotypes sig-nificantly decreased on the first day of cold exposure and then showed a recovery until day 7 . WS- 1 was not detected in the leaves of either genotype. Withanone (WS-2) content in the leaves also decreased towards the end of the cold period in both genotypes. Cold stress also elicited the accumulation of WS-2 in AGB025, but was not detectable in control seedlings. At maturity, WS-2 was also detected in control plants. Furthermore, a significant increase in the leaf withaferin A (WS-3) content was recorded from day 1 to day 7 of the cold exposure in both the genotypes, suggesting the possible involvement of with-anolides in cold-protection. AGB002 showed comparatively higher accumulation of antioxidant enzymes and selected marker withanolides than AGB025, indicating that AGB002 is better adapted to cold than AGB025. It could be inferred from these observations that cold stress induces bioactive withanolide accu-mulation in $W$. somnifera as a mechanism for scavenging reactive oxygen species (ROS). These studies also provide an impetus for enhancing the withanolide accumulation in $W$. somnifera using controlled environment technology.
\end{abstract}

\section{Keywords:}

Withania somnifera Cold

stress

Reactive oxygen species

Withanolides

Anti-oxidant enzymes

\footnotetext{
Abbreviations: APX, ascorbate peroxidase (EC 1.11.1.11); BSA, bovine serum albumin; CAT, catalase (EC 1.11.1.6); DCPIP, 2,6-dichlorophenolindophenol; DTT, dithiothreitol; EDTA, ethylenediaminetetraacetic acid; GR, glutathione reductase (EC 1.6.4.2); GSH, reduced glutathione; GSSG, oxidized glutathione; $\mathrm{H}_{2} \mathrm{O}_{2}$, hydrogen peroxide; HPLC, high performance liquid chromatography; MDA, malondialdehyde; MTT, 3-(4,5-dimethyl-2-thiazolyl)-2,5-diphenyl-2H-tetrazolium bromide; NBT, nitro blue tetrazolium; $\mathrm{O}_{2}{ }^{-}$-, superoxide radicals; PAGE, polyacrylamide gel electrophoresis; ROS, reactive oxygen species; SOD, superoxide dismutase (EC 1.15.1.1); TBA, thiobarbutyric acid; TCA, trichloroacetic acid; TEMED, N,N,N',N-tetramethylethylenediamine; TTC, triphenyl tetrazolium chloride; WS-1, withanolide A; WS-2, withanone; WS-3, withaferin A; PMSF, phenylmethylsulfonyl fluoride; PVPP, polyvinylpolypyrrolidone; DTNB, 5,5'-dithiobis-(2-nitrobenzoic acid).

* Corresponding author at: Centre for Microbial Ecology and Genomics \& Genomics Research Institute, Department of Genetics, University of Pretoria, Pretoria 0028, South Africa.
}

E-mail address: meerbilal82@gmail.com (B.A. Mir). 


\section{Introduction}

Plants are very often exposed to a combination of different kinds of biotic and abiotic stresses rather than a single stress in their natural environment. These environmental stresses, irrespective of source, trigger numerous plant responses, ranging from altered gene expression and cellular metabolism to changes in plant biochemistry, growth and development (Miller et al., 2008; Jaleel, 2009 Ahmad et al., 2010; Huang et al., 2012; Mishra et al., 2014). Cold stress, one of the most harmful abiotic stresses affects growth, development and geographic distribution of plants. Induction of the lipid peroxidation in plants due to excessive generation of reactive oxygen species (ROS) is one major consequence of cold and other abiotic stresses (Mittler, 2002; Mahajan and Tuteja, 2005; Miller et al., 2008; Gill and Tuteja, 2010; Ahmed et al., 2010 Mutlu et al., 2013). ROS molecules include free radicals such as superoxide $\left(\mathrm{O}_{2}{ }^{-}\right)$, hydroxyl radical $(-\mathrm{OH})$ and non radical molecules like hydrogen peroxide $\left(\mathrm{H}_{2} \mathrm{O}_{2}\right)$ and singlet oxygen $\left({ }^{1} \mathrm{O}_{2}\right)$. At low concentrations, ROS act as signaling molecules, however, when produced in excess these molecules have toxic effects. They react with cellular macromolecules (proteins, lipids and/or DNA) causing oxidative damage and inactivation of metabolic enzymes leading to impaired cell functions (Mittler, 2002; Mishra and Gupta, 2006Tuteja and Sopory, 2008 Gill and Tuteja, 2010; Huang et al., 2012).

Plants have evolved several enzymatic and non-enzymatic mechanisms to provide protection against the toxic effects of ROS and to maintain cellular redox homeostasis (Ahmad et al., 2012; Jaleel et al., 2007, 2009; Kaur et al., 2012; Sabir et al., 2012; Mishra et al., 2014). ROS scavenging enzymes include catalase (CAT), ascorbate peroxidase (APX), superoxide dismutase (SOD), and glutathione reductase (GR; Noctor and Foyer, 1998). The enzymatic components of ascorbate-glutathione cycle (APX, MDHAR, DHAR and GR), the major ROS scavenging pathways utilize ascorbic acid and glutathione to scavenge $\mathrm{H}_{2} \mathrm{O}_{2}$ (Asada, 1999; Ahmad et al., 2010). GR catalyzes the reduction of glutathione disulfide (GSSG) to reduced glutathione (GSH) with the accompanying oxidation of NADPH. SOD converts superoxide radicals to $\mathrm{H}_{2} \mathrm{O}_{2}$, while CAT, found in peroxisomes, breaks down $\mathrm{H}_{2} \mathrm{O}_{2}$ into water and molecular oxygen (Scandalios et al., 1997; Polidoros and Scandalio, 1999). Based on the metal cofactor they require SODs are classified into manganese (Mn-SOD), iron (Fe-SOD) and copper/ zinc (Cu/Zn-SOD) (Mittler, 2002). A concerted and balanced action of antioxidant enzymes is required to functionalize their scavenging systems and mechanisms.

The ability of the plants to minimize oxidative stress depends upon the effectiveness of enzymatic and non-enzymatic antioxidants (Blokhina et al., 2003; Seppanen and Coleman, 2003). The antioxidant potential is highly variable in different plant species and in different genotypes of the same species. Stress tolerant genotypes have often been reported to show higher expression of antioxidants as reported for salt (Walia et al., 2005), drought (Türkan et al., 2005), cold (Seppanen and Coleman, 2003,b; Kumar et al., 2008a,b; Mutlu et al., 2013) and heat stress (Snider et al., 2010; Almeselmani et al., 2009). The responsiveness and efficiency of scavenging systems of plants governs their performance, endurance, and survival capability under stress conditions (Seppanen and Coleman, 2003; Mishra et al., 2014).

Withania somnifera (commonly known as Indian ginseng) belongs to the family Solanaceae and is a medicinal plant of substantial therapeutic value and wide geographic distribution. It has received considerable attention throughout the world due to its potential preventive and curative role in several clinical problems including neurodegeneration, in the treatment of tumours and in its use as an antihelminthic (Sangwan et al., 2004; Tuli et al., 2009; Yang et al., 2011; Sehgal et al., 2012). The medicinal properties of $W$. somnifera have been attributed to withanolides synthesized in the leaves and roots. In recent years, there is an increasing demand of these bioactive withanolides in pharmaceutical industry (Mir et al., 2012, 2014a). However, the composition and quality of withanolides shows great variability in plants growing in different environmental conditions (Kumar et al., 2011; Mir et al., 2014a).

Plants interact with their environment by producing a diverse array of secondary metabolites. The physiological, biochemical and molecular changes caused by temperature stress can alter the secondary metabolites concentrations in the plant tissues, and are often used as an indicator of stress injury in the plant. An appropriate thermoperiod is considered to be a key factor in shaping the secondary metabolite composition and quality of medicinal plants. In $W$. somnifera seasonal low temperature has been reported to play an important role in increasing the accumulation of bioactive withanolides (Kumar et al., 2012). The objective of the present study was to investigate the effect of cold stress $\left(4^{\circ} \mathrm{C}\right)$ on lipid peroxidation, cell injury, superoxide radical accumulation, antioxidant enzyme activities and accumulation of three marker withanolides (WS-1, WS-2 and WS-3) in two contrasting genotypes/morphochemovariants (AGB002 and AGB025) of $W$. somnifera.

\section{Methods and materials}

\subsection{Plant materials}

Two morphochemovariants (AGB002 and AGB025) of W. somnifera were selected for the present study. AGB002 (an elite selectant originating from Rajasthan, India) is rich in root biomass associated withanolide A, whereas leaves of AGB025 (an elite selectant from Madhya Pradesh, India, now termed W. ashwagandha) are rich in withaferin A. Freshly harvested seeds of both these accessions growing in the experimental field of Indian Institute of Integrative Medicine (IIIM), Jammu ( $32^{\circ} 44^{\prime} \mathrm{N}$ latitude and $75^{\circ} 55^{\prime}$ E longitude at an altitude of approx. $400 \mathrm{~m}$ a.s.l) were germinated in earthen pots in controlled greenhouse conditions $\left(25^{\circ} \mathrm{C}, 16 \mathrm{hr}\right.$ photoperiod and $75 \%$ air humidity).

\subsection{Imposition of cold stress}

Two month old seedlings were exposed to cold stress at $4{ }^{\circ} \mathrm{C}$ for seven days in a plant growth chamber. Plants maintained at $25^{\circ} \mathrm{C}$ in the green house were used as controls. Young leaves (same leaf position) and roots from the control and cold stressed plants were harvested at each stage. Harvesting was done at 1, 3, 5 and 7 days after cold exposure and analyzed for proteins content, lipid peroxidation (MDA), cell injury, superoxide radical accumulation and anti-oxidative enzymes activities (SOD, CAT, APX and GR) and secondary metabolites (WS-1, WS-2 and WS-3). Some plants exposed to cold elicitation were shifted to control green house conditions and grown to maturity. At maturity, both controls and cold stressed plants were analyzed for the three marker withanolides.

\subsection{Quantification of cell injury}

The Triphenyl tetrazolium chloride (TTC) reduction test was used to estimate cold injury in leaves exposed to cold stress (Vyas and Kumar, 2005). Control and cold-exposed leaf discs (50 mg) were suspended in $1.5 \mathrm{ml}$ of $0.8 \%$ TTC $(\mathrm{w} / \mathrm{v})$ solution prepared in $50 \mathrm{mM}$ phosphate buffer ( $\mathrm{pH}$ 7.4) followed by vacuum infiltration for $15 \mathrm{~min}$. Following the dark incubation of $16 \mathrm{~h}$ at $25^{\circ} \mathrm{C}$, leaf discs were washed with distilled water and re-suspended in $95 \%$ ethanol. The test tubes were placed in a boiling water bath for $5 \mathrm{~min}$ and the reduced formazan was extracted after the final volume was made 
up to $1.5 \mathrm{ml}$ using $95 \%$ ethanol. The absorbance was recorded at $530 \mathrm{~nm}$.

\subsection{Superoxide $\left(\mathrm{O}_{2}{ }^{-}\right)$estimation}

For superoxide radical estimation leaf discs were vacuum infiltrated in $1.5 \mathrm{ml}$ of reaction mixture $(50 \mathrm{mM}$ phosphate buffer, $\mathrm{pH}$ 7.8 and NBT, $5 \mathrm{mg} / \mathrm{ml}$ ). The samples (reaction mixture containing leaf discs) were boiled for $15 \mathrm{~min}$ following the incubation of $30 \mathrm{~min}$ at $25^{\circ} \mathrm{C}$. The insoluble formazan produced by the reduction of NBT was estimated according to the method of Vyas et al. (2007). The formazan produced was pelleted by centrifugation, dissolved in $1 \mathrm{ml}$ of $N, N$-dimethylformamide and measured spectrophotometrically at $580 \mathrm{~nm}$. For control experiments leaf discs were boiled before incubation in the reaction mixture.

\subsection{Lipid peroxidation assay}

Lipid peroxidation was determined using the method of Heath and Packer (1968). For the measurement of malondialdehyde (MDA), $1 \mathrm{~g}$ leaf tissues were homogenized with a mortar and pestle in $1 \mathrm{ml}$ of $0.5 \%$ trichloroacetic acid (TCA) and centrifuged for $20 \mathrm{~min}$ at $14,000 \mathrm{rpm}$. Half of the supernatant was mixed with a solution containing $2.5 \mathrm{ml}$ of $20 \%$ TCA and $0.5 \%$ thiobarbutyric acid. The solution was then heated for $30 \mathrm{~min}$ at $95^{\circ} \mathrm{C}$ and subsequently paced in an ice bath for cooling. After centrifugation of the solution for $10 \mathrm{~min}$ at $10,000 \mathrm{rpm}$, the resulting supernatant was used for determination of MDA content by recording the absorbance at $532 \mathrm{~nm}$. The MDA equivalent was calculated as follows; MDA (nmol/ml $F W)=\{($ A532-A600) $/ 155,000\} \times 106$, where $F W=$ fresh weight and $\mathrm{A}=$ absorbance.

\subsection{Extraction and antioxidant enzyme activity assay}

For extraction of enzymes and the estimation of protein content, $100 \mathrm{mg}$ sample of leaf tissues from third leaf position were ground to powder in liquid nitrogen and lysed in $1 \mathrm{ml}$ pre-chilled extraction buffer (50 mM phosphate buffer, pH 7.8, 2 mM EDTA, 1 mM DTT, $1 \mathrm{mM}$ PMSF and $0.5 \%(\mathrm{v} / \mathrm{v})$ Triton X-100) at $4{ }^{\circ} \mathrm{C}$. PVPP $(10 \% \mathrm{w} / \mathrm{v})$ was added before grinding to absorb the polyphenols. The supernatant of the homogenate centrifuged for $20 \mathrm{~min}$ at $15,000 \times \mathrm{g}$ at $4{ }^{\circ} \mathrm{C}$ was collected for protein estimation and enzyme assays. Protein content was estimated according to the Bradford (1976) assay using bovine serum albumin (BSA, Sigma) as standard. For CAT and APX estimation, phosphate buffer of $\mathrm{pH} 7$ and 7.2 was used, with the addition of $3 \mathrm{mM}$ ascorbate in the latter. For the estimation of GR, oxidized glutathione (GSSG; $3 \mathrm{mM}$ ) was added to the extraction buffer.

\subsubsection{Superoxide dismutase activity}

Superoxide dismutase (SOD; EC 1.15.1.1) activity was determined spectrophotometrically at $560 \mathrm{~nm}$ (Vyas et al., 2007). The reaction mixture containing $20 \mu \mathrm{l}$ tissue extract, $2 \mu \mathrm{M}$ riboflavin, $50 \mu \mathrm{M}$ NBT, $10 \mathrm{mM}$ DL-methionine and $0.025 \%$ Triton X-100 in a potassium phosphate buffer $(50 \mathrm{mM}$; $\mathrm{pH} 7.8$ ) was illuminated with a cool light source for $5 \mathrm{~min}$ at $25^{\circ} \mathrm{C}$. The absorbance of the formazan produced was recorded at $560 \mathrm{~nm}$.

\subsubsection{Ascorbate peroxidase activity}

Ascorbate peroxidase (APX; EC 1.11.1.11) activity assays were performed at $25^{\circ} \mathrm{C}$ in potassium phosphate buffer $(50 \mathrm{mM}$; pH 7.0) containing $0.1 \mathrm{mM}$ EDTA, $0.5 \mathrm{mM}$ ascorbate and $2 \mathrm{mM} \mathrm{H} \mathrm{H}_{2}$ (Nakano and Asada, 1981). Reactions were initiated by addition of $10 \mu \mathrm{l}$ of the tissue extract in $1 \mathrm{ml}$ reaction buffer. Ascorbate oxidation was monitored continuously for $5 \mathrm{~min}$ by measuring the decrease in the absorbance at $290 \mathrm{~nm}\left(\epsilon=2.8 \mathrm{mM}^{-1} \mathrm{~cm}^{-1}\right)$. APX was expressed in units $(\mathrm{U}) / \mathrm{mg}$ protein. One $\mathrm{U}$ is defined as the change in the absorbance by $0.1 / \mathrm{min} / \mathrm{mg}$ protein.

\subsubsection{Glutathione reductase activity}

Glutathione reductase (GR; EC 1.6.4.2) activity assays were performed at RT in $50 \mathrm{mM}$ potassium phosphate buffer ( $\mathrm{pH} 7.8$ ) following the method of Jahnke et al. (1991). Tissue extract $(20 \mu \mathrm{l})$ placed in a buffer containing $1 \mathrm{mM}$ GSSG, $1 \mathrm{mM}$ EDTA and $0.1 \mathrm{mM}$ NADPH was incubated at $37^{\circ} \mathrm{C}$ for $30 \mathrm{~min}$. The decrease in the absorbance at $412 \mathrm{~nm}$ was continuously monitored $(\epsilon$ $\mathrm{NADPH}=6.22 \mathrm{mM}^{-1} \mathrm{~cm}^{-1}$ ). GR was expressed in units where one unit is defined as the change in the absorbance of $0.1 / \mathrm{min} / \mathrm{mg}$ protein.

\subsubsection{Catalase activity}

Catalase (CAT) assays were performed in potassium phosphate buffer ( $50 \mathrm{mM}$; pH 7.0) supplemented with $20 \mathrm{mM} \mathrm{H} \mathrm{H}_{2}$. CAT activity was measured by monitoring $\mathrm{H}_{2} \mathrm{O}_{2}\left(\epsilon=39.4 \mathrm{mM}^{-1} \mathrm{~cm}^{-1}\right)$ consumption (Sood et al., 2006). In a quartz cuvette reaction was initiated by adding enzyme extract $(10 \mu \mathrm{l})$ and decrease in absorbance was continuously monitored for $5 \mathrm{~min}$ at $240 \mathrm{~nm}$. CAT activity was measured by monitoring the consumption of following the method of. Control reactions for each of the enzymes assayed were carried out using boiled enzyme extract.

\subsection{SDS-PAGE, Native PAGE and activity staining (in-gel assay)}

Tissue extracted proteins were visualized by native polyacrylamide gel electrophoresis (PAGE), using 10\% resolving gels and $4 \%$ stacking gels. SOD activity was visualized by incubating the gel in $50 \mathrm{mM}$ phosphate buffer ( $\mathrm{pH} 7.8$ ) containing $0.3 \mathrm{mM}$ NBT and $30 \mu \mathrm{M}$ riboflavin for $30 \mathrm{~min}$ in the dark and then immersing the gel in $28 \mathrm{mM}$ TEMED followed by exposure to a light source at RT (Beauchamp and Fridovich, 1971). GR isoforms were detected by incubating the gels in $50 \mathrm{mM}$ Tris- $\mathrm{HCl}(\mathrm{pH} 7.8)$ containing $1.5 \mathrm{mM}$ NADPH, $4.0 \mathrm{mM}$ oxidized glutathione (GSSG) and $2 \mathrm{mM}$ DTNB for $30 \mathrm{~min}$ in the dark and visualized by incubating the gel in buffer containing $1.5 \mathrm{mM}$ MTT and $1.6 \mathrm{mM}$ PMS for $10 \mathrm{~min}$ at room temperature. For detection of APX, gels were incubated for $30 \mathrm{~min}$ in potassium phosphate buffer ( $50 \mathrm{mM}$; pH 7.0) containing $4 \mathrm{mM}$ ascorbate followed by an incubation of $20 \mathrm{~min}$ in the buffer containing $6 \mathrm{mM}$ ascorbate and $2.0 \mathrm{mM} \mathrm{H}_{2} \mathrm{O}_{2}$. After washing with buffer for $2 \mathrm{~min}$, the gels were submerged in a solution of phosphate buffer ( $\mathrm{pH} 7.8$ ) added with $30 \mathrm{mM}$ TEMED and $3 \mathrm{mM}$ NBT for the visualization of isoforms. For CAT activity, gels were rinsed with double distilled water and then incubated in a solution of $\mathrm{H}_{2} \mathrm{O}_{2}(0.010 \%$; $\mathrm{v} / \mathrm{v}$ ) for $20 \mathrm{~min}$. Following a brief rinse with water, the gels were incubated in $1 \%$ ferric chloride and $1 \%$ potassium ferricyanide solution for $15 \mathrm{~min}$, respectively for detection of CAT bands (Woodbury et al., 1971).

\subsection{HPLC quantification of withanolides}

Withanolides (withanolide A, withanone and withaferin A) were quantified using HPLC-based procedures as described previously (Kumar et al., 2011; Mir et al., 2014b). Standards prepared in HPLCgrade methanol were used to identify and quantify the analytes in the tissue extracts. Analyses were performed with a Waters HPLC system (Waters, Milford, MA). Samples for HPLC analysis were also filtered through $0.45 \mu \mathrm{M}$ filter (Millipore, Bedford, MA). Extracts were separated using RP 18 (Merck, Bangalore, India) column. Samples were eluted using a solution of HPLC grade water and HPLC grade methanol prepared in the ratio of 60:40. The flow rate was set at $0.7 \mathrm{ml} \mathrm{min}^{-1}$. Samples were analyzed at $30^{\circ} \mathrm{C}$ and the absorbance 
was monitored at $237 \mathrm{~nm}$. Values were expressed in terms of $\mathrm{mg} / \mathrm{g}$ dry weight tissue of withanolides.

\subsection{Statistical analysis}

All the assays were performed with three replicates $(n=3)$ and data were expressed as mean values \pm SE. Statistical analyses were performed by analysis of variance (ANOVA) and means were compared by Duncan's multiple range test at $P<0.05$.

\section{Results and discussion}

This study was conducted in $W$. somnifera to understand the accumulation/induction and relationship of marker bioactive withanolides (WS-1, WS-2 and WS-3) and anti-oxidative defense system on exposure to cold stress. Initially $W$. somnifera seedlings were exposed to cold stress for two weeks but the seedlings after day 8 showed complete necrosis of the plants, hence, seven day cold exposure has been selected for the present study.

\subsection{Cell damage and oxidative stress}

Our results on cell damage clearly showed that on the first day of exposure to cold stress both the genotypes (AGB002 and AGB025) showed a significant $(P \leq 0.05)$ cellular damage as assessed by TTC reduction test (Fig. 1a). The cellular damage increased up to day 7 in both genotypes; however, AGB002 exhibited 15\% lesser damage and showed good recovery on day 7 as compared to AGB025. This lesser cellular damage in AGB002 compared to AGB025 suggested a relatively efficient protection mechanism in AGB002. Vyas and Kumar (2005) also reported that some tea clones depicted better antioxidant system and are better adapted to cold. This reduction in cell viability in both the accessions on exposure to cold stress may be attributed to the accumulation of ROS that cause oxidative damage to lipids, proteins and nucleic acids (Imlay, 2003; Vyas and Kumar, 2005; Huang et al., 2012).

Formation of MDA used to assess the level of lipid peroxidation serves as a measure of damage primarily due to ROS induced by cold stress. On prolonged cold stress up to day 7 in this study MDA production was observed to increase significantly $(P \leq 0.05)$ in AGB025 (Fig. 1b). The intensity of lipid peroxidation in AGB002 showed a different trend as it significantly increased from up to third day of the cold exposure and then decreased abruptly to the level of control toward the end of the cold period (Fig. 1b). The recovery towards the end of the cold exposure in AGB002 may be due to the increased expression of antioxidants. AGB025 exhibited significantly higher lipid peroxidation ( $50 \%$ ) than AGB002 (Fig. 1b). Increase in lipid peroxidation under stress conditions was earlier reported in $W$. somnifera (Sabir et al., 2012) and Swertia cherata (Abrol et al., 2012). Increased lipid peroxidation under light and cold stress was also obtained by Shohael et al. (2006) in Eleutherococcus senticosus.

In order to understand the correlation between increase in lipid peroxidation and ROS accumulation under cold stress (Kocsy et al., $1997,2001)$, we measured superoxide radical $\left(\mathrm{O}_{2}{ }^{-}\right)$concentration during the course of experiment. Significant increase in superoxide ions from day 1 to day 7 of the cold stress in both the genotypes was observed except until day 5 in case of AGB025 (Fig. 1c). In AGB002 the increase of $\mathrm{O}_{2}{ }^{-}$radicals was $102 \%$ compared to control plants on day 7 of the experiment whereas, in AGB025, $\mathrm{O}_{2}{ }^{-}$increased by $140 \%$ compared to control plants. It was interesting to observe the correlation between $\mathrm{O}_{2}{ }^{-}$- and MDA concentration in AGB025 which was in agreement with the previous studies of Kocsy et al. (1997, $2001)$,). The reduction in peroxidation and cell viability towards the end of the cold exposure observed in AGB002 may be due to the better antioxidant enzyme machinery. The present data support the contention that cold stress induced generation of ROS may


Fig. 1. Effect of cold stress on the on cell viability/changes in TTC reduction (A), lipid peroxidation (B), superoxide anion content $(C)$ in two genotypes of $W$. somnifera. Values are mean of three independent replicates $\pm \mathrm{SD} ;\left(^{*}\right)$ represents the significance values at $(P \leq 0.05)$; $C$, control that are grown in green house condition.

be responsible for cellular damage and induction of antioxidant enzymes (Kendall and Mckersie, 1989; Kuroda and Sagisaka, 1992; Walker and McKersie, 1993; Vyas and Kumar, 2005).

\subsection{Antioxidant enzymes}

Antioxidant enzymes are effective quenchers of ROS and their activation help plants to maintain cellular homeostasis during stressful environment. Activities of SOD, GR, APX and CAT in both genotypes increased significantly $(P \leq 0.05)$ as compared to control plants (Fig. 2). Relative increase in the activities of SOD, GR, APX and CAT in AGB002 on day 7 was higher than AGB025 (Fig. 2). Increase in the SOD activities under cold stress has been reported in previous studies (Seppänen and Fagerstedt, 2000; Sahoo et al., 2001 Vyas et al., 2007). Of the $\mathrm{H}_{2} \mathrm{O}_{2}$ scavenging enzymes, both APX and CAT induction starts from the first day and its levels increased significantly towards the end of cold exposure, whereas (Fig. 2c and d). The increased activity and expression of antioxidant enzymes and levels of antioxidant molecules in $W$. somnifera and other plant 
A $120-\quad \begin{aligned} & \mathrm{AGB} 002 \\ & \mathrm{AGB025}\end{aligned}$
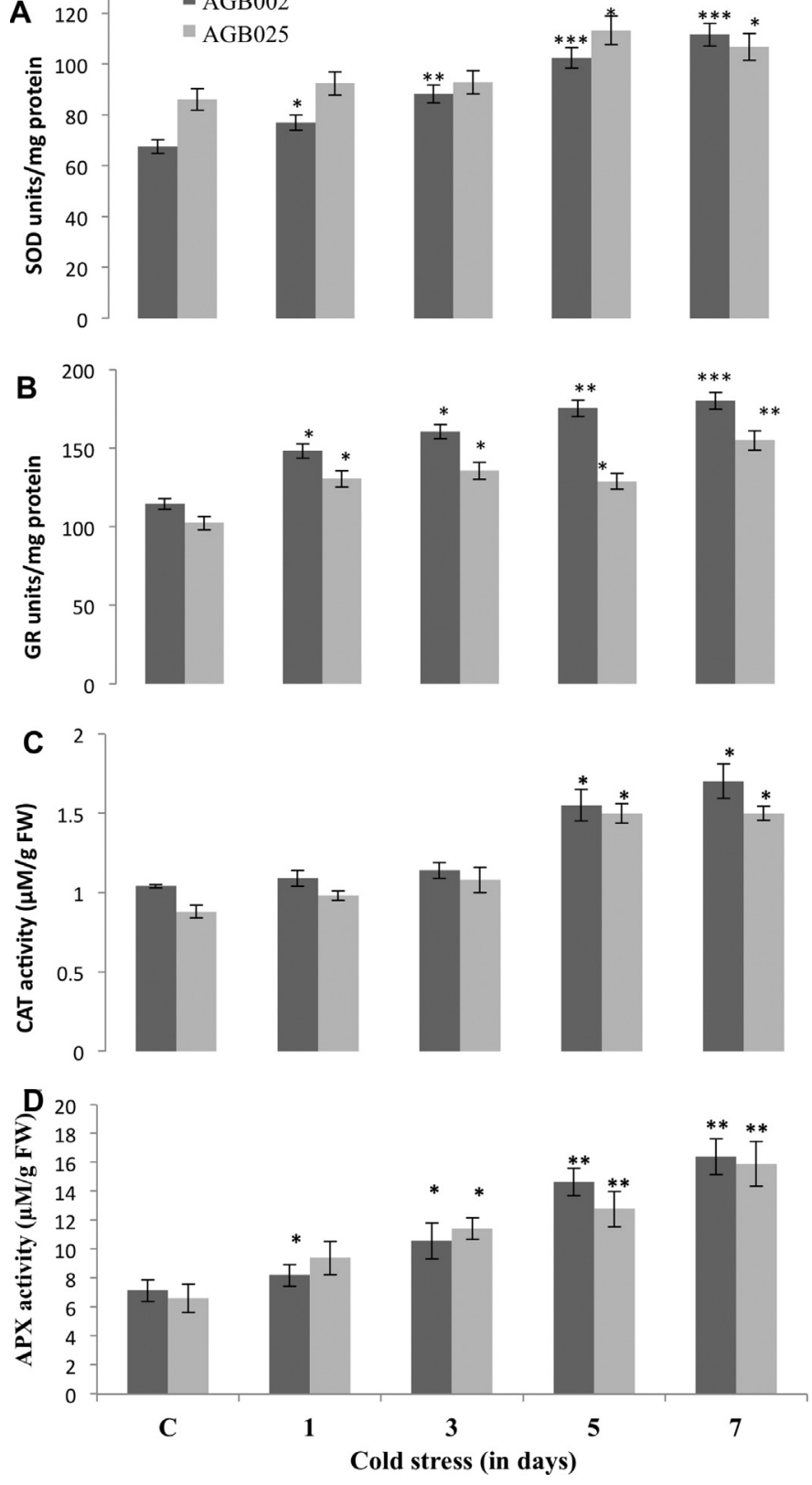

Fig. 2. Changes in antioxidant enzyme activities of AGB002 and AGB025 genotypes of W. somnifera after cold stress. SOD activity (A), GR activity (B), CAT activity (C) and APX activity (D). Values are mean of three independent replicates \pm SD and $\left(^{*}\right)$ represents the significant values.

species under various stresses has also been reported earlier (Kocsy et al., 1996, 2001; Jaleel et al., 2008, 2009; Kaur et al., 2012; Sabir et al., 2012; Mishra et al., 2014). The combined action of CAT and SOD converts the $\mathrm{O}^{2-}$ and $\mathrm{H}_{2} \mathrm{O}_{2}$ to water and $\mathrm{O}_{2}$, thus, prevent the cellular damage under unfavorable condition (Arora et al., 2014). GR plays an important role in maintaining the cellular redox status, especially during low temperature induced photo-inhibition (Noctor and Foyer, 1998; Kocsy et al., 2001). Greater increase in GR would certainly help in maintaining a higher GSH/GSSG ratio, which is essential in ensuring the correct folding and appropriate activity of the proteins and redox signaling of various defense genes (Polidoros and Scandalios, 1999). High GR activities have been shown in various chilling tolerant genotypes (Walker and McKersie, 1993; Kocsy et al., 1996). It is quite likely that increased levels of GR keep glutathione in the reduced state and play a sig- nificant role in protecting cellular homeostasis against cold stress damage in $W$. somnifera. Further, higher activities of antioxidant enzymes suggested a role of these enzymes in imparting better protection to $W$. somnifera under cold temperature.

The different forms of antioxidant enzymes analyzed on native polyacrylamide gel showed quantitative (increase in band intensity) differences in response to cold period and the expression was higher on 7th day of the cold stress when compared to control plants in both the accessions (Fig. 3). The isoforms of SOD (three), GR (three), APX (two) and CAT (one) were observed in native PAGE (Fig. 3). The staining area/band intensity of the two bands of APX was almost twice in cold exposed plants compared to the control one (Fig. 3b and f). Similar results were seen earlier in some other plant materials by Mittler (2002) and Scandalios (2005). Similarly, the enzyme activities of GR and APX showed increased band intensity in both the genotypes with slightly increased intensity of bands observed in AGB002 than AGB025. Up-regulation of APX was also reported in rice seedlings exposed to cold stress (Chen et al., 2012). It was interesting to note the presence and up-regulation of three isofoms of SOD under the experimental condition (Fig. 3a and e). That these enzymes help to combat the ROS in oxidative stress is indicated by a reduction in the oxidative stress towards the end of cold period after these enzymes are elicited and their expression reached to maximum. Empirical evidence is available for a positive correlation between increase in SOD activity and decrease in oxidative stress as reported in the present study (Huang et al., 2012; Sabir et al., 2012; Mishra et al., 2014).

Genotypic variation observed in the present study suggested differential efficiencies of antioxidant mechanisms are operative in $W$. somnifera during cold stress. The ability of the plants to minimize the oxidative stress depends upon the effectiveness of the antioxidants, which has been reported to vary according to the plant species and their genotypes (Blokhina et al., 2003; Seppanen and Coleman, 2003). From these results it is clear that AGB002 showed superior expression of antioxidant enzymes on exposure to cold stress than ABG025. These results are in close agreement with that of Kumar et al. (2008a,b; Kumar et al. (2008a,b) who also reported that cold-stress tolerant genotypes possess superior expression of antioxidants enzymes.

\subsection{Effect of cold on withanolides}

Abiotic stresses including various elicitors or signal molecules in plants often result in the increased accumulation of secondary metabolites, the main sources for pharmaceuticals, flavors, and industrially important biochemicals. A representative HPLC chromatogram of extract prepared from root tissue is shown in Fig. 4. Our results showed that accumulation of all three selected marker withanolides were altered substantially both in roots and leaves of AGB002 and AGB025 on exposure to cold stress (Fig. 5a-f). In the roots of both genotypes the content of withanolide A (WS1) first decreased significantly from day 1 to day 3 of the cold exposure and then sharply increased up to day 7. Withaferin A (WS-3) content of roots also decreased from day 1 to day 7 of cold treatment compared to the control plants (Fig. 5a-b and e-f). Withanone (WS-2) in the roots increased sharply on day 1 of the cold stress and thereafter decreased up to day 5 and then again it showed a recovery (Fig. $5 \mathrm{c}$ and d). The increased concentration of these withanolides after prolonged cold stress of 7 days may be possibly due to the increased accumulation and expression of antioxidant enzymes indicative of positive correlation between withanolides and antioxidant enzymes. As observed in the present study secondary metabolites have previously been reported to play a major role in the adaptation of plants to the cold and in overcoming other stressful environments conditions (Kliebenstein, 2004; Ramakrishna and Ravishankar, 2011). 

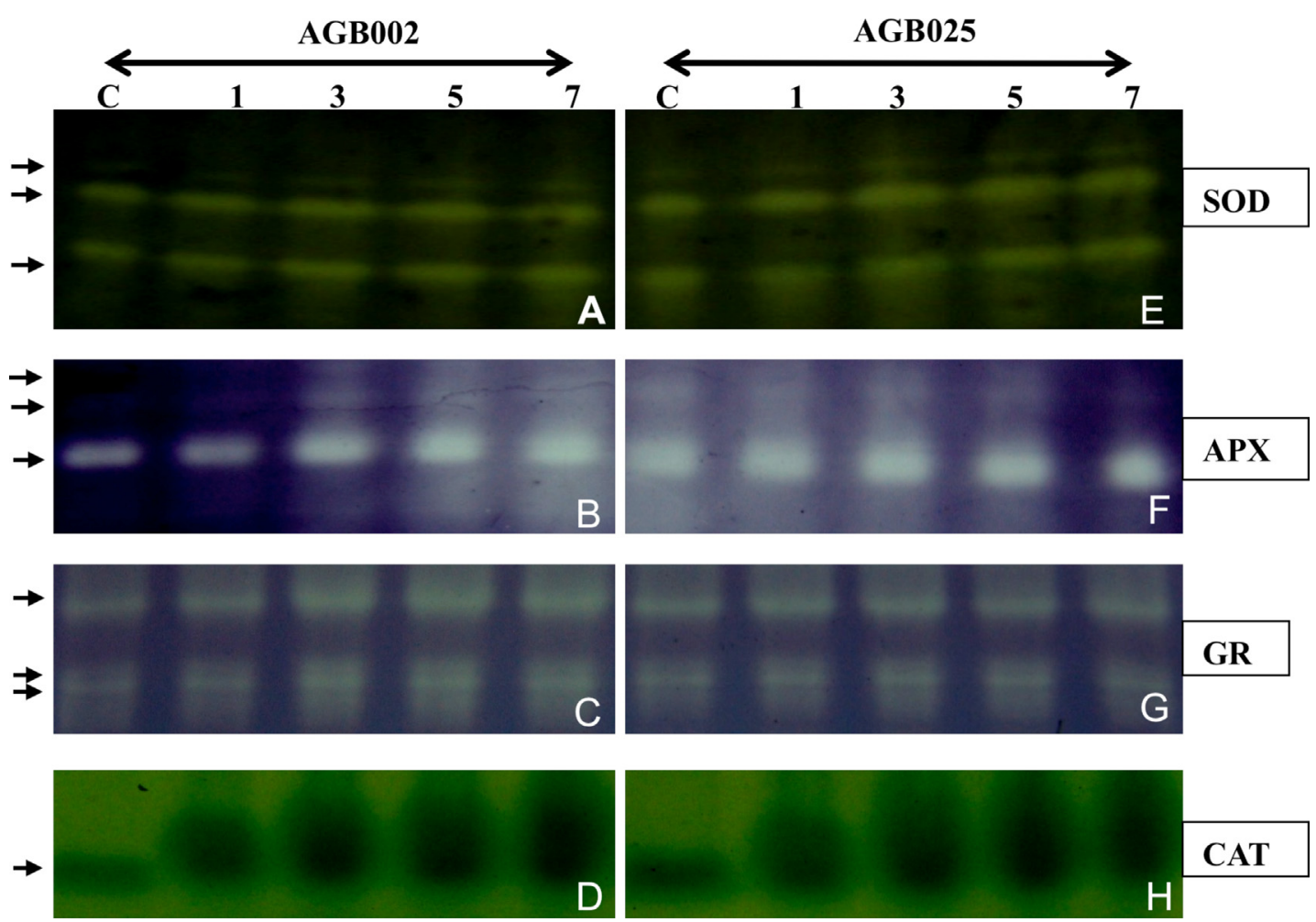

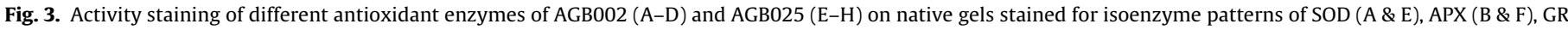

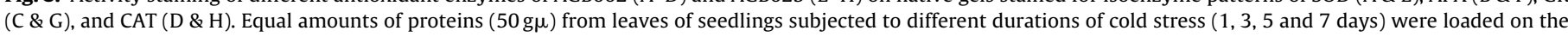

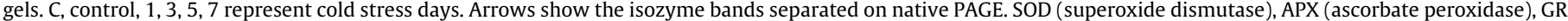
(glutathione reductase); CAT (catalase). $\left({ }^{*}\right)$ represents the significance values at $(P \leq 0.05)$.

Interestingly, in leaves (the direct targets of cold stress) marker withanolides followed a different trend. WS-1 was not detected in the leaves of both the genotypes. WS-2 in both the genotypes decreased from day 1 to day 7 of the cold exposure (Fig. $5 c$ and d). However, WS-3 increased constantly from day 1 to day 7 in the leaves of both genotypes indicating positive effect of cold stress on WS-3 elicitation (Fig. 5e and f). Increase in withanolide contents might be due to the increased regulation of large number of transcripts including withanolide metabolic pathway genes. Previous studies have also reported positive correlation between biotic/abiotic stress and secondary metabolite accumulation (Kirakosyan et al., 2004; Wahid and Ghazanfar 2006; Cheng et al., 2007Ghorpade et al., 2011 Abrol et al., 2012). Recently, Ahire et al. (2014) also reported enhanced accumulation of bacoside $A$ in Bacopa monnieri exposed to salt stress under controlled environmental conditions. The authors repot that this increase in the accumulation of active principle may be due to increased level of $\mathrm{H}_{2} \mathrm{O}_{2}$ caused by lipid peroxidation that functions as a signal molecule for the induction of plant defense systems including the enhanced secondary metabolite production (Ahire et al., 2014).WS2 in the leaves of AGB025 was either absent or below detection level in control plants but was detected in the plants on the first day of exposure to cold stress (Fig. 4d). Kumar et al. (2012) also reported that seasonal low temperature increases withanolide accumulation due to metabolic shift in W. somnifera. Withanolides may have a role in combating oxidative stress in the leaves of $W$. somnifera. The

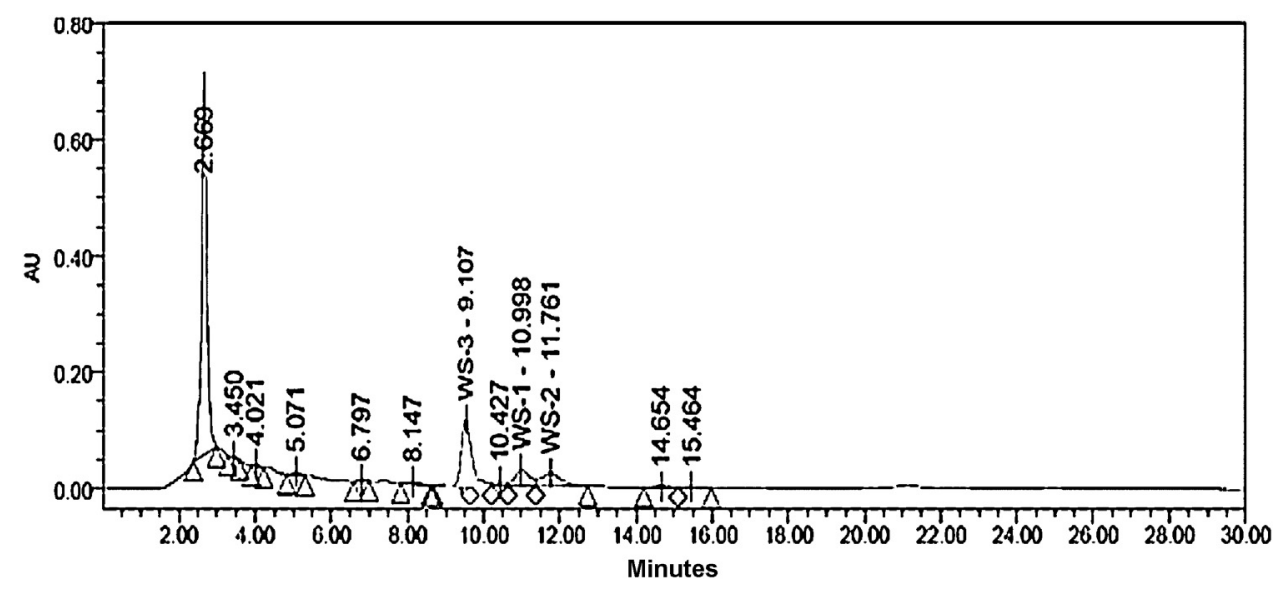

Fig. 4. Representative chromatogram of leaf extract; WS-1 (withanolide A); WS-2 (withanone); and WS-3 (withaferin A). 

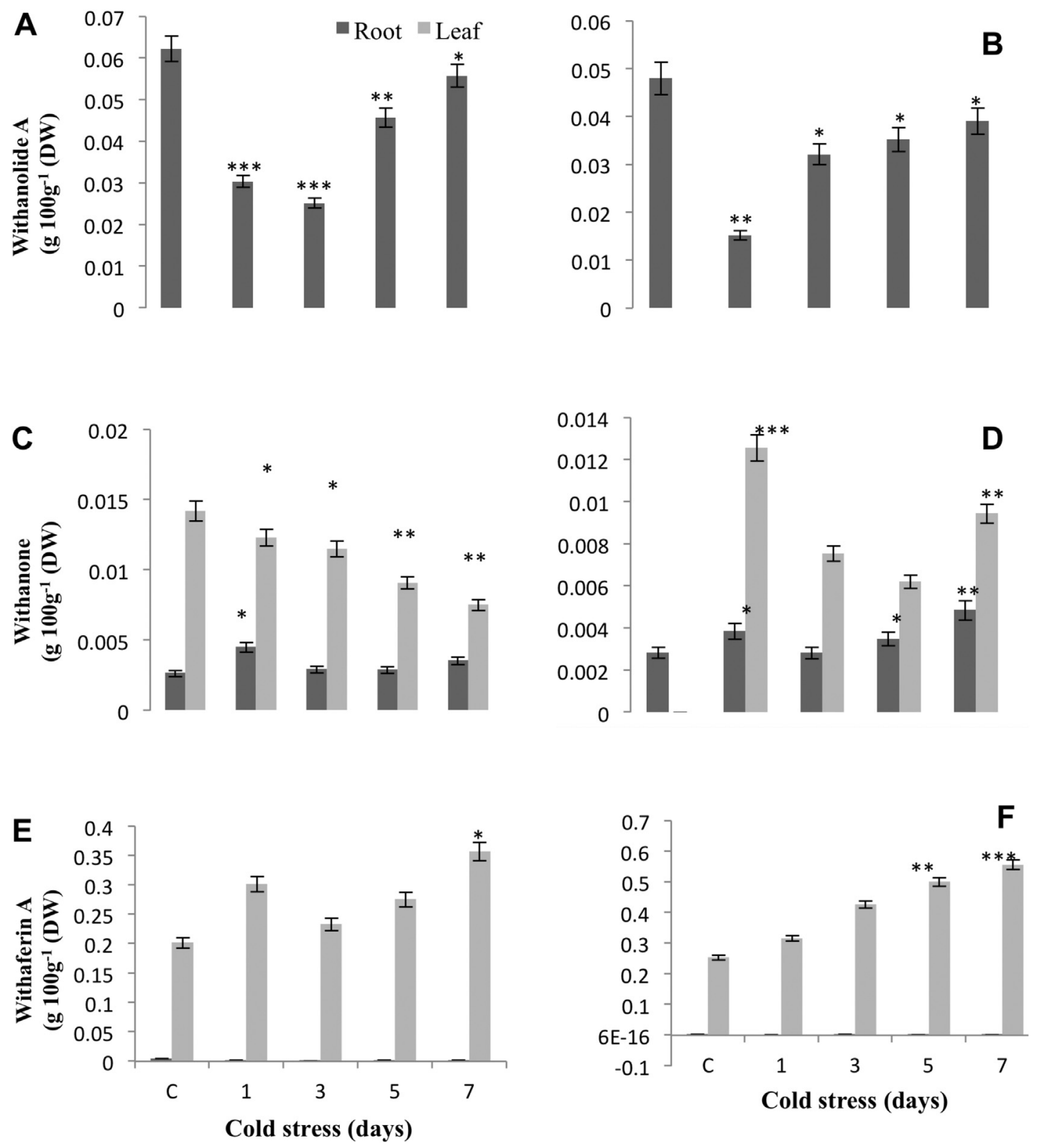

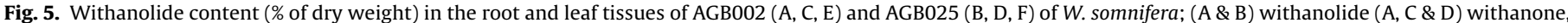

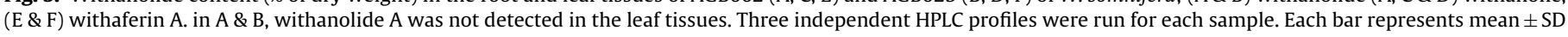
of three independent replicates.

regulation of plant secondary metabolites and their positive correlation with antioxidant potential under various abiotic stresses has also been demonstrated in Ocimum sanctum (Selvam et al., 2013).

After continuous cold stress for seven days five plants were shifted to green house and grown alongside with control plants till maturity stage and analyzed for three marker withanolides. It was observed that cold stressed plants showed higher accumulation of withaferin A than control plants (Fig. 6). WS-2 was also detected in AGB025 at the maturity stage suggesting that cold stress induces its expression (data not shown). The reduction in peroxidation towards the end of the cold exposure probably due to the increased expression of antioxidant enzymes might be involved in the upregulation of secondary metabolite production in $W$. somnifera. These results provide the first indication that low temperature is an important environmental factor for optimization of secondary metabolite production in $W$. somnifera and controlled environment

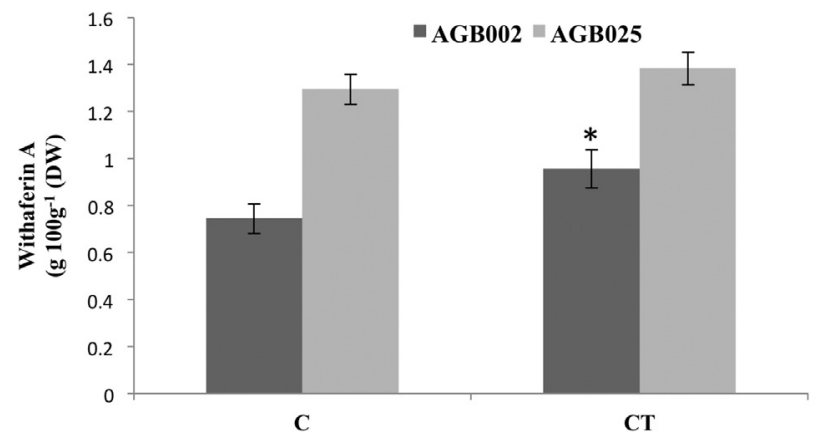

Fig. 6. Withaferin A content in the leaf tissues of AGB002 and AGB025 of W. somnifera quantified at maturity stage; $C$, control plants grown in green house, and CT, cold treated plants that were grown in green house after cold treatment for seven days. Each bar represents mean \pm SD of three independent replicates. 
technology can allow the precise application of the cold stress. Further studies such as the effect of various withanolides on the antioxidant defense system and gene expression studies to understand the target proteins that are disregulated are needed.

\section{Conclusion}

In conclusion, a significant increase in the anti-oxidative enzymes activities (SOD, CAT, APX and GR) and the contents of marker withanolides was observed after seven days of cold treatment. The findings presented here demonstrated a positive relationship between induction of antioxidant enzymes and production of withanolides in $W$. somnifera under the experimental conditions. Based on our results it appears that AGB002 experienced lesser oxidative stress in response to cold treatment as compared to AGB025 and can be cultivated under varied environmental conditions. Our studies also showed that short interval of cold treatment is sufficient to invoke elicitation of withanolides in $W$. somnifera. The results also showed that interplay of enzymatic as well as non-enzymatic antioxidative responses together with elicitation of withanolides constituted a system endeavor of tolerance of cold stress imposition and an efficient scavenging strategy of toxic free radicals allowing the plant to grow under cold conditions. The plant resorts to modulate its metabolism in such a way to avoid the destructive effect of oxidative stress and evolves a biochemical machinery to detoxify cold-generated toxicity. More detailed studies are needed to elucidate the mechanisms of cold-induced responses related to medicinally important withanolides. Further, understanding the detoxification mechanism and strategies in $W$. somnifera against the cold stress at plant level will provide insights into manipulating cold stress tolerance in medicinally important plant.

\section{Acknowledgements}

Authors gratefully acknowledge CSIR-Indian Institute of Integrative Medicine (CSIR-IIIM), Jammu for the fellowship and facilities to carry this work. The assistance, support and guidance of Dr. Arun Kumar (Senior Technical Officer, CSIR-IIIM) are highly acknowledged. Thanks are also due to Mr. Prabu Datt, Natural Product Chemistry Division, CSIR-IIIM for his technical help in HPLC.

\section{References}

Abrol, E., Vyas, D., Koul, S., 2012. Metabolic shift from secondary metabolite production to induction of anti-oxidative enzymes during $\mathrm{NaCl}$ stress in Swertia chirata Buch. Ham. Acta Physiol. Plant 34, 541-546.

Ahire, M.L., Laxmi, S., Walunj, S.R., Kavikishor, P.B., Nikam, T.D., 2014. Effect of potassium chloride and calcium chloride induced stress on in vitro cultures of Ba. J. Plant Biochem. Biotechnol. 23, 366-378.

Ahmad, P., Jaleel, C.A., Salem, M.A., Nabi, G., Sharma, S., 2010. Roles of enzymatic and non-enzymatic antioxidants in plants during abiotic stress. Crit. Rev. Biotechnol. 30, 161-175.

Asada, K., 1999. The water-water cycle in chloroplasts: scavenging of active oxygens and dissipation of excess photons. Annu. Rev. Plant Physiol. Plant Mol. Biol. 50, 601-639.

Beauchamp, C., Fridovich, I., 1971. Superoxide dismutase: improved assays and an assay applicable to acrylamide gels. Anal. Biochem. 44, 276-287.

Blokhina, O., Violainen, E., Fagerstedt, K.V., 2003. Antioxidants, oxidative damage and oxygen deprivation stress: a review. Ann. Bot. 91, 179-194.

Bradford, M.M., 1976. A rapid and sensitive method for the quantitation of microgram quantities of protein utilizing the principle of protein dye binding. Ann. Biochem. 72, 248-253.

Gill, S.S., Tuteja, N., 2010. Reactive oxygen species and antioxidant machinery in abiotic stress tolerance in crop plants. Plant Physiol. Biochem. 48, 909-930.

Heath, R.L., Packer, L., 1968. Photoperoxidation in isolated chloroplasts. I. Kinetics and stoichiometry of fatty acid peroxidation. Arch. Biochem. Biophys. 125, 189-198.

Huang, G.T., Ma, S.L., Bai, L.P., Zhang, L., Ma, H., Jia, P., Liu, J., Zhong, M., Guo, Z.F. 2012. Signal transduction during cold, salt, and drought stresses in plants. Mol. Biol. Rep. 39, 969-987.

Imlay, J.A., 2003. Pathways of oxidative damage. Annu. Rev. Microbiol. 57, 395-418.
Jahnke, L.S., Hull, M.R., Long, S.P., 1991. Chilling stress and oxygen metabolizing enzymes in Zea mays and Zea diploperennis. Plant Cell Environ. 14, 97-104.

Jaleel, C.A., Gopi, R., Manivannan, P., Panneerselvam, R., 2008. Exogenous application of triadimefon affects the antioxidant defense system of Withanid somnifera Dunal. Pest. Biochem. Physiol. 91, 170-174.

Jaleel, C.A., Manivannan, P., Lakshmanan, G.M.A., Sridharan, R., Panneerselvam, R., 2007. $\mathrm{NaCl}$ as a physiological modulator of proline metabolism and antioxidant potential in Phyllanthus amarus. CR Biol. 330, 806-813.

Jaleel, C.A., Riadh, K., Gopi, R., Manivannan, P., Ines, J., Al-Juburi, H.J., Xing, Z.C., Bo, S.H., Panneerselvam, R., 2009. Antioxidant defense responses: physiological plasticity in higher plants under abiotic constraints. Acta Physiol. Plant 31, 427-436.

Kaur, G., Singh, H.P., Batish, D.R., Kohil, R.K., 2012. A time course assessment of changes in reactive oxygen species generation and antioxidant defense in hydroponically grown wheat in response to lead ions $\left(\mathrm{Pb}^{+2}\right)$. Protoplasma 249 , 1091-1100.

Kendall, E.J., Mckersie, B.D., 1989. Free radical and freezing injury to cell membranes of winter wheat. Physiol. Plant. 76, 86-94.

Kirakosyan, A., Sirvent, T., Gibson, D., Kaufman, P., 2004. The production of hypericins and hyperforin by in vitro cultures of Hypericum perforatum Biotechnol. App. Biochem. 39, 71-81.

Kliebenstein, D.J., 2004. Secondary metabolites and plant/environment interactions: a view through Arabidopsis thaliana tinged glasses. Plant Cell Environ. 27, 675-684.

Kocsy, G., Brunner, M., Rüegsegger, A., Stamp, P., Brunold, C., 1996. Glutathione synthesis in maize genotypes with different sensitivities to chilling. Planta 198 365-370.

Kocsy, G., Galiba, G., Brunold, C., 2001. Role of glutathione in adaptation and signaling during chilling and cold acclimation in plants. Physiol. Plant. 113, $158-164$.

Kocsy, G., Owttrim, G., Brander, K., Brunold, C., 1997. Effect of chilling on the diurnal rhythm of enzymes involved in protection against oxidative stress in a chilling-tolerant and a chilling-sensitive maize genotype. Physiol. Plant. 99, 249-254.

Kumar, A., Abrol, E., Koul, S., Vyas, D., 2012. Seasonal low temperature plays an important role in increasing metabolic content of secondary metabolites in Withania somnifera (L.) Dunal and affects the time of harvesting. Acta Physiol Plant 34, 2027-2031.

Kumar, A., Bali, Y., Sharma, K.D., Thakral, S.K., 2008a. Evaluation of wheat genotypes for terminal heat tolerance by simple physiological traits. Ind. J. Plant Physiol. 13, 21-27

Kumar, S., Kaur, G., Nayyar, H., 2008b. Exogenous application of abscisic acid improves cold tolerance in chickpea (Cicer arietinum L.). J. Agron. Crop Sci. 194, 449-456.

Kumar, A., Mir, M.A., Sehgal, D., Dar, T.H., Koul, S., Kaul, M.K., Raina, S.N., Qazi, G.N., 2011. Utility of multidisciplinary approach for genome diagnostics of cultivated and wild germplasm resources of medicinal Withania somnifera, and status of new species, W. ashwagandha, in the cultivated taxon. Plant Sys. Evol. 291, 141-151.

Kuroda, H., Sagisaka, S., 1992. Malfunction of enzyme systems involved in the regeneration of glutathione in perennials at low temperature. Biosci. Biotech. Biochem. 56, 712-715.

Mahajan, S., Tuteja, N., 2005. Cold, salinity and drought stresses: an overview. Arch. Biochem. Biophys. 444, 139-158.

Miller, G., Shulaev, V., Mittler, R., 2008. Reactive oxygen signaling and abiotic stress. Physiol. Plant. 133, 481-489.

Mir, B.A., Khazir, J., Hakeem, K.R., Koul, S., Cowan, D.A., 2014a. Enhanced production of withaferin-A in shoot cultures of Withania somnifera (L) Dunal. J. Plant Biochem. Biotechnol. 23, 430-434.

Mir, B.A., Khazir, J., Hakeem, K.R., Kumar, A., Koul, S., 2014b. Withanolides array of Withania ashwagandha sp. novo populations from India. Ind. Crops Prod. 59, 9-13.

Mishra, B., Sangwan, R.S., Mishra, S., Jadaun, J.S., Sabir, F., 2014. Effect of cadmium stress on inductive enzymatic and non-enzymatic responses of ros and sugar metabolism in multiple shoot cultures of Ashwagandha (Withania somnifera Dunal). Protoplasma 251, 1031-1045.

Mittler, R., 2002. Oxidative stress, antioxidants and stress tolerance. Trends Plant Sci 7, 405-410.

Mutlu, S., Karadağoğlu, O., Atici, O., Nalbantoglu, B., 2013. Protective role of salicylic acid applied before cold stress on antioxidative system and protein patterns in barley apoplast. Biol. Plant 57, 507-513.

Nakano, Y., Asada, K., 1981. Hydrogen peroxide is scavenged by ascorbate-specific peroxidase in spinach chloroplasts. Plant Cell Physiol. 22, 867-880.

Noctor, G., Foyer, C.H., 1998. Ascorbate and glutathione: keeping active oxygen under control. Annu. Rev. Plant Physiol. Plant Mol. Biol. 49, 249-279.

Polidoros, A.N., Scandalios, J.G., 1999. Role of hydrogen peroxide and different classes of antioxidants in the regulation of catalase and glutathione S-transferase gene expression in maize (Zea mays L.). Physiol. Plant. 106, 112-120.

Ramakrishna, A., Ravishankar, G.A., 2011. Influence of abiotic stress signals on secondary metabolites in plants. Plant Signal. Behav. 6, 1720-1731.

Sabir, F., Sangwan, R.S., Kumar, R., Sangwan, N.S., 2012. Salt stress-induced responses in growth and metabolism in callus cultures and differentiating in vitro shoots of Indian ginseng (Withania somnifera Dunal). J. Plant Growth Regul. 31, 537-548. 
Sangwan, R.S., Chaurasiya, N.D., Mishra, L.N., Lal, P., Uniyal, G.C., Sharma, R., Sangwan, N.S., Suri, K.A., Qazi, G.N., Tuli, R., 2004. Phytochemical variability in commercial herbal products and preparations of Withania somnifera (Ashwagandha). Curr. Sci. 86, 461-465.

Scandalios, J.G., 2005. Oxidative stress: molecular perception and transduction of signals triggering antioxidant gene defenses. Braz. J. Med. Biol. Res. 38, 995-1014.

Scandalios, J.G., Guan, J., Polidoros, A.N., 1997. Catalases in plants: gene structure, properties regulation and expression. In: Scandalios, J.G. (Ed.), Oxidative Stress and the Molecular Biology of Antioxidant Defenses. Cold Spring Harbor Laboratory, Cold Spring Harbor, New York, USA, pp. 343-406.

Sehgal, N., Gupta, A., Khader, R., Shanker, V., Joshi, D., Mills, J.T., Hamel, E., Khanna P., Jain, S.C., Thakur, S.S., Ravindranath, V., 2012. Withania somnifera reverses Alzheimer's disease pathology by enhancing low-density lipoprotein receptor-related protein in liver. PNAS 109, 3510-3515.

Selvam, K., Rajinikanth, R., Govarthanan, M., Paul, A., Selvankumar, T., Sengottaiyan, A., 2013. Antioxidant potential and secondary metabolites in Ocimum sanctum L. at various habitats. J. Med. Plants Res. 7, 706-712.

Seppänen, M.M., Fagerstedt, K., 2000. The role of superoxide dismutase in response to cold acclimation in potato. Physiol. Plant. 108, 279-285.

Seppanen, M.M., Coleman, G.D., 2003. Characterization of genotypic variation in stress gene expression and photosynthetic parameters in potato. Plant Cell Environ. 26, 401-410.

Sood, S., Vyas, D., Nagar, P.K., 2006. Physiological and biochemical studies during flower development in two rose species. Sci. Hortic 108, 390-396.

Tuli, R., Sangwan, R.S., Kumar, S., Bhattacharya, S., Misra, L.N., Mandal, C., Raghubir, R., Trivedi, P.K., Tewari, S.K., Mishra, P., Chaturvedi, P., Sangwan, N.S., Nair, K.N.,
Rawat, A.K.S., Srivastava, V., Srivastava, R.K., Ojha, S.K., Mehrotra, S., Khajuria, A., Suri, K.A., 2009. Ashwagandha (Withania somnifera) A Model Indian Medicinal Plant. In: Tuli, R., Sangwan, R.S. (Eds.). NMITLI-CSIR, New Delhi, pp. 978-993, ISBN No.978-93-80235-29-5.

Türkan, I., Bor, M., Ozdemir, F., Koca, H., 2005. Differential responses of lipid peroxidation and antioxidants in the leaves of drought-tolerant $P$. acutifolius Gray and drought-sensitive P. vulgaris L. subjected to polyethylene glycol mediated water stress. Plant Sci. 168, 223-231.

Vyas, D., Kumar, S., 2005. Tea (Camellia sinensis (L.) O. Kuntze) clone with lowe period of winter dormancy exhibits lesser cellular damage in response to low temperature. Plant Physiol. Biochem. 43, 383-388.

Vyas, D., Kumar, S., Ahuja, P.S., 2007. Tea clones with shorter periods of winter dormancy exhibit lower accumulation of reactive oxygen species. Tree Physiol 27, 1253-1259.

Wahid, A., Ghazanfar, A., 2006. Possible involvement of some secondary metabolites in salt tolerance of sugarcane. J. Plant Physiol. 163, 723-730.

Walia, H., Wilson, C., Condamine, P., et al., 2005. Comparative transcriptional profiling of two contrasting rice genotypes under salinity stress during the vegetative growth stage. Plant Physiol. 139, 822-835.

Walker, M., McKersie, B., 1993. Role of the ascorbate-glutathione antioxidant system in chilling resistance of tomato. Plant Physiol. 141, 234-239.

Woodbury, W., Spencer, A.K., Stahman, M.A., 1971. An improved procedure using ferricyanide for detecting catalase isozymes. Anal. Biochem. 44, 301-305.

Yang, E.S., Choi, M.J., Kim, J.H., Choi, K.S., Kwon, T.K., 2011. Withaferin A enhances radiation-induced apoptosis in Caki cells through induction of reactive oxygen species, Bcl-2 down regulation and Akt inhibition. Chem. Biol. Interact. 190, $9-15$ 